\title{
Additivity of Heats of Combustion, LCAO Resonance Energies, and Bond Orders of Conformal Sets of Conjugated Compounds*
}

\author{
R. A. Marcus \\ Noyes Chemical Laboratory, University of Illinois, Urbana, Illinois
}

(Received 12 May 1965)

\begin{abstract}
The notion of "conformal sets" of aromatic and other conjugated compounds is introduced. To a good approximation, it is found that the sum of the heats of combustion of the compounds in one set equals the sum for the compounds in the other set. Similar additivity occurs for their LCAO-calculated total $\pi$-electron energies, LCAO bond orders and free valences. Bond lengths and dissociation energies are also considered. Conformal sets are defined in terms of their number and quality of self-returning random walks on the atoms of the conjugated compounds. Even-electron nonalternant and alternant compounds are included, as are, in certain cases, radicals. Some insight into the additivity for LCAO properties is obtained by relating the coefficients in LCAO secular equations to random walks and using contour integral formulas connecting LCAO properties with secular determinants.
\end{abstract}

\section{INTRODUCTION}

$\mathbf{I}^{\mathrm{N}}$ $\mathrm{N}$ the present paper the notion of conformal sets is introduced. The concept does not involve bond energies but can be usefully supplemented by nearestneighbor bond concepts, as described later. We find that to a reasonable approximation the sum of the heats of combustion of a set of compounds equals the sum of that of another set which conforms to the first set, geometrically or otherwise, by satisfying certain conditions. We also find that a similar additivity exists for LCAO $\pi$-electron energies. (Thus, an additivity of heats of combustion does not in itself imply that the chemical bonding is localized.)

The additivity applies to conjugated molecules containing heteroatoms, to alternant and nonalternant compounds, and to radicals conforming to certain conditions. Hence, it is exhibited both by compounds having only one classical structure and for which the resonance concept has been questioned ${ }^{1}$ and by compounds for which an observed additivity is somewhat more surprising. It is shown that an additivity reported for certain aromatics ${ }^{2 \mathrm{a}}$ is a special instance of conformal-set additivity. Additivity was also known previously in other formats for some of the compounds (compare tables of "group contributions" such as those of six-membered rings, etc. ${ }^{2 b}$ ).

Conformal sets are defined here in terms of random walks. Some insight into the additivity in delocalized (LCAO) systems is obtained by relating the coefficients

\footnotetext{
* Supported by a grant from the National Science Foundation. A portion of the research was performed during the tenure of an Alfred P. Sloan Fellowship.

${ }_{1}$ M. J. S. Dewar and G. J. Gleicher, J. Am. Chem. Soc. 87, 692, 685 (1965) ; M. J. S. Dewar and H. N. Schmeising, Tetrahedron 11, $96(1960) ; 5,166(1959) ;$ A. L. H. Chung and M. J. S. Dewar, J. Chem. Phys. 42, 756 (1965).

${ }^{2}$ (a) C. J. McGinn, Tetrahedron 18, 311 (1962); (b) G. J. Janz, Estimation of Thermodynamic Properties of Organic Compounds (Academic Press Inc., New York, 1958), Chap. 4, which contains references to previous work.
}

of the secular determinant to random walks and by using a contour-integral formula connecting $\pi$-electron energy and secular determinants.

\section{CONFORMAL SETS}

We define a self-returning random walk in a molecule as a walk from neighboring atom to neighboring atom, which begins and ends on the same atom and during which one may pass a particular atom once or several times. Two walks on different compounds are said to have the same quality if the atoms at corresponding steps are chemically similar. ${ }^{3}$

Each conformal set consists of one or more aromatic or other conjugated compounds. The two sets conform to each other if they satisfy the following conditions:

(1) Each compound present has an even number of electrons. ${ }^{5}$

(2) The number and quality of two-step, four-step, five-step and, when greater accuracy is desired, sixstep self-returning random walks in one set equal the number and quality in the other set.

${ }^{3}$ More precisely, by chemical similarity of these corresponding atoms we mean that they are atoms of the same element and are in fairly similar nearest-neighbor environments. For example, the carbon atom in the 9 position in naphthalene is regarded as chemically similar in this sense to the atom in the 11 position in anthracene. Each carbon atom in benzene is regarded as chemically similar to the carbon atoms in the 1 and 2 positions in naphthalene.

${ }^{4}$ Compounds with three-step walks are tentatively omitted from the definition: Three-step walks arise from circumnavigation of a three-membered ring, and the experimental data on these rings are presently limited.

${ }^{5}$ An anion may contribute to one set, if it fulfills Conditions (1) to (3), if another anion contributes to the other set, and if the local ionic charge-density function is approximately the same in two anions. The latter condition is added to assure similar quality walks in actual molecules, but not in the simplest LCAO calculations where all atoms are made identical regardless of molecular charge. However, we have not tested conformality for actual anions as yet. 
(3) No compound has an LCAO eigenvalue of zero. ${ }^{6}$ This condition can also be phrased in non-LCAO terms. ${ }^{7}$

Recognition of (2) can often be accomplished (up to four steps and occasionally up to six) by appropriate superposition of compounds in one set and comparison with superposed compounds in a second set (Appendix I). There are instances, however, where conformality up to six steps is desirable ${ }^{8}$ and where it is not recognized by this simple geometric method. Partly for this reason the definition of conformality was not based on superposition. ${ }^{8 b}$ For such cases, and in general, a simple way of counting random walks by counting numbers of different bonds and rings is derived later.

Condition (3) is a major one since it reflects large behavioral differences of some compounds that would otherwise satisfy (1) and (2). ${ }^{9}$ In some, but not all, instances the excluded compounds are those whose classical structures are radicallike in the uncharged state (e.g., $m$-quinone).

${ }^{B}$ This condition becomes unimportant for very large molecules. The difference between large molecules with eigenvalues close to zero and those with zero eigenvalues becomes small with the increasing size. For example, cyclic polyenes with $4 n+2$ carbon atoms are aromatic but, experimental data and SCF-MO calculations indicate, cease to be aromatic when $n$ is about 6 or 7 (See Ref. 1, Dewar and Gleicher, p. 685.)

${ }^{7}$ Condition (3) can be rephrased as follows:

(3a) There is at least one way of connecting adjacent atoms in the conjugated part of each compound by islands of polygons so that no atom is left unconnected. One or more of these polygons may be degenerate, i.e., may consist of a straight line connecting two adjacent atoms.

(3b) A sum obtained by the following recipe shall not vanish: In all arrangements of disjointed polygons in (3a) one assigns a value of 1 to one consisting of degenerate polygons only and a value of $2^{n}$ to one containing $n$ nondegenerate polygons; one then gives a sign to each term in the latter by assigning $(-1)^{m+1}$ to each polygon of $m$ vertices in that term and multiplying the latter signs; and finally one sums over all terms.

Fulfillment of Property (3a) can readily be recognized. oQuinone and $p$-quinone fulfill (3a): The islands of polygons here consist of four straight lines which coincide with the double bonds in the classical structure of the molecules in this case. An example of a compound that does not fulfill (3a) is $m$-quinone. Here, two atoms are left unconnected by the islands of polygons. The cyclopentadienyl radical does not fulfill (1) but fulfills (3a): The "islands of polygons" here consist of one island, a single pentagon. The anion of cyclopentadienyl fulfills both (1) and (3).

Normally, one can assume that if (3a) is fulfilled so is (3b). There are some rare cancellations, to be sure, and then $(3 \mathrm{~b})$ is not fulfilled but (3a) is: In a cyclic polyene containing $4 n$ atoms, for example, the sum in $(3 \mathrm{~b})$ is $-2+(-1)(-1)+(-1)(-1)=0$.

${ }^{8}$ (a) Consider, for example, the series naphthacene, benz (a) anthracene, chrysene, and triphenylene in Table VI. From the results in Tables $V$ and VI these compounds are seen to be four-step but not six-step conformal. They show a systematic change in heats of combustion $\left(2170.6,2169.8,2165.0\right.$, and $2164.4 \mathrm{kcal} \mathrm{mole}^{-1}$, respectively), in LCAO energies $(-24.93,-25.10,-25.19$, $-25.27 \beta$, respectively ) and in numbers of six-step walks. 3-4 Benzophenanthrene is six-step conformal to chrysene and has an LCAO energy of $-25.19 \beta$. It is omitted from Table I, since the molecule is overcrowded. Its heat of combustion is $2172.5 \mathrm{kcal}$ mole $^{-1}$. (b) Several examples where six-step conformality is not recognizable by superposition but is recognizable by suitable bond and ring count are given later.

${ }^{9}$ The unsynthesized $m$-quinone has an LCAO zero eigenvalue but $o$ - and $p$-quinones do not. Cyclic polyenes with $4 n$ conjugated carbon atoms have zero eigenvalues but those with $4 n+2$ atoms do not; the latter are aromatic. Linear conjugated systems with an odd number of carbon atoms have zero eigenvalues but those with an even number do not.
An extension of the above definition to sets which contain certain radicals ("weakly conformal sets") and to sets which almost have the same number of four-step walks ("nearly conformal") is given later.

TABLE I. Heats of combustion of conformal sets of conjugated compounds."

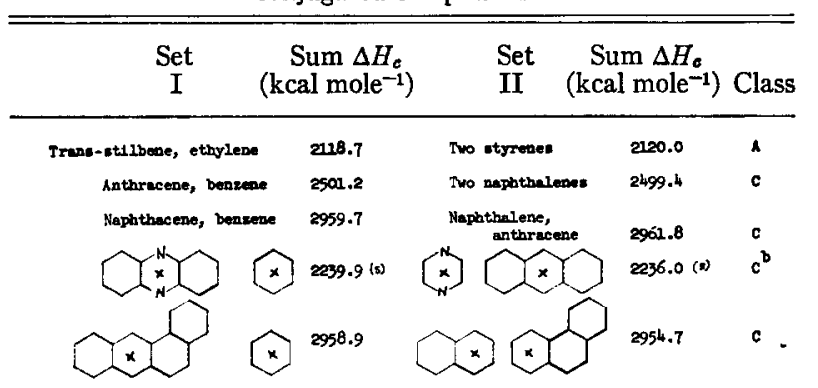

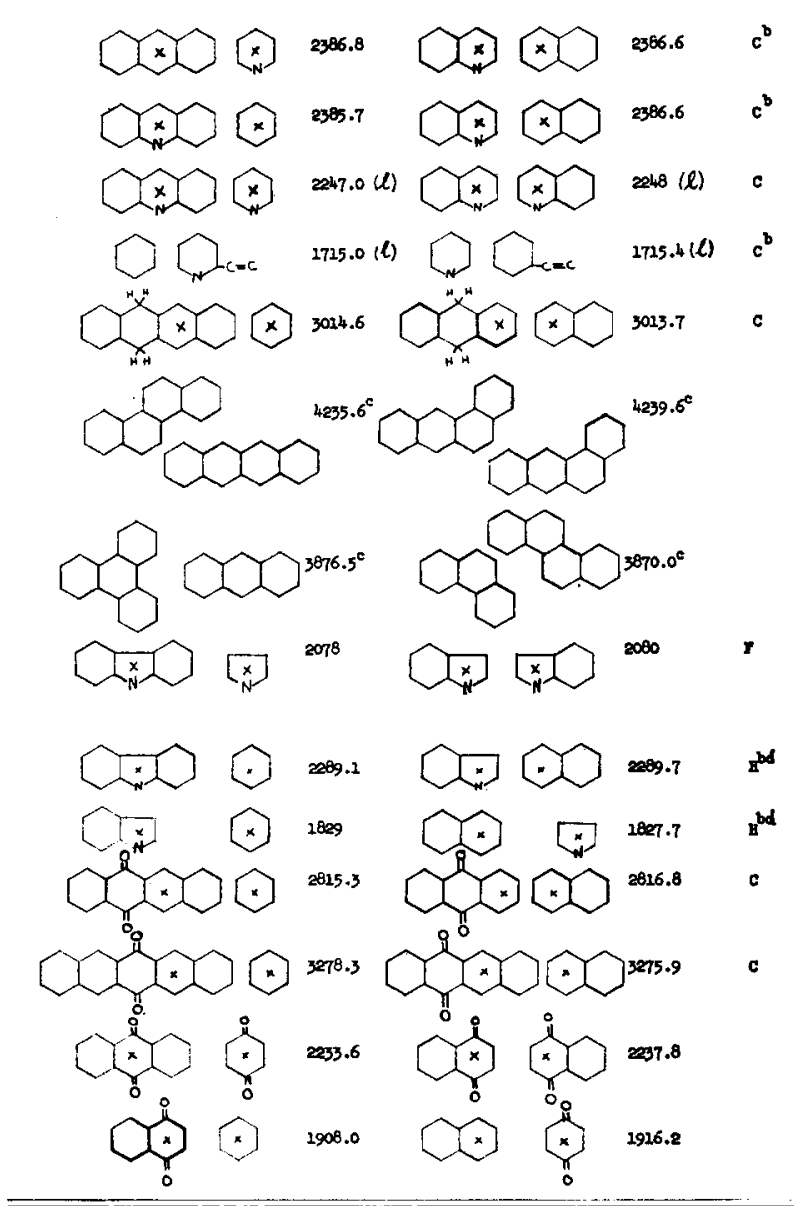

a All data refer to gases, except where indicated by $s$ (solid) or 1 (liquid). The x's indicate rings to be superposed. All rings shown, except one in the tenth row involving $4 \mathrm{H}$ 's, are aromatic. The $\mathrm{N}$ in each five-membered ring is presumed to contribute two electrons to the ring. Class $C$ is six-step conformal. The remaining classes are four-step conformal.

${ }^{b}$ Quality of a few of the numerous four-step walks differs in the two sets, since $\mathrm{N}$ differs from $\mathrm{C}$.

- For comparison with some of the other figures, the difference of sums $\Delta B_{c}$ should be divided by a factor of about 2 to place the comparison on the same percentage basis. The examples have six-step conformality.

d Quality of a few of the numerous four-step walks differs in the two sets, since a five-ring bond differs from a six-ring bond. 


\section{DATA ON CONFORMALITY}

Experimental data ${ }^{10}$ are given in Table I and LCAO $\pi$-electron energies ${ }^{11}$ in Table II. These tables contain no examples where the sets differ in nonconjugated groups (except for Eample 10 in Table I), since the

TABLE II. Sum of theoretical LCAO $\pi$-electron energies $E$ for conformal sets."

(16) sersatriene, ethylene (units of $\beta$ ) Set II

a Resonance energies are obtained by adding to $+E$ the number in parentheses at the beginaing of each row. The $x$ 's indicate the rings that are to be superposed.

10 (a) Data are largely taken from G. W. Wheland, Resonance in Organic Chemistry (John Wiley \& Sons, Inc., New York, 1955). (b) Sources of data for other compounds are as follows: (quinones) A. Magnus, H. Hartmann, and F. Becker, Z. Physik. Chem. (Leipzig) 197, 75 (1951); (phenazine and acridine). J. B. Willis, Trans. Faraday Soc. 43, 97 (1947); (2-vinyl pyridine) A. A. Balandin, E. I. Klabunovskii, A. P. Oberemok-Yakubova, and I. I. Brusov, Izvest. Akad. Nauk SSSR Otd. Khim. Nauk 1960, 748 [Chem. Abstr. 56, 10998i (1962) ]; (pyrazine) J. Tjebbes, Acta Chem. Scand. 16, 916 (1962); (styrene, l) E. J. Prosen, R. Gilmont, and F. D. Rossini, J. Res. Natl. Bur. Std. 34, 65 (1945).

is Data are taken from LCAO calculations given in Dictionary of Values of Molecular Constants, edited by C. A. Coulson and R. Daudel (Mathematical Institute, Oxford, England and Centre de Mécanique Ondulatoire Appliquée, Paris, post 1959, undated),

Vols. II and III. Some data were taken from references cited therein. additivity for such groups is regarded as established. The x's in these tables indicate superposition, not structural detail.

The deviation between sums of $E$ in Table II is usually no more than about 0.06 , i.e., about 0.03 per compound, and often less. A difference of $0.03 \beta$ corresponds to about $0.6 \mathrm{kcal} \mathrm{mole}^{-1}$. The additivity of energies in Tables I and II is perhaps all the more striking when one compares individual LCAO eigenvalues in the conformal sets, as in the following one, for example: anthracene, benzene, $\epsilon / \beta=-2.41,-2.00$, $-2.00,-1.41,-1.41,-1.00,-1.00,-1.00,-1.00$, -0.41 ; two naphthalenes, $\epsilon / \beta=-2.30,-2.30,-1.62$, $-1.62,-1.30,-1.30,-1.00,-1.00,-0.62,-0.62$. For this reason LCAO properties of the individual orbital energies, spectral transition energies for example, are not considered in the present paper.

\section{BROADER DEFINITION WHICH INCLUDES WEAK CONFORMALITY}

Sets which satisfy the following definition but not the earlier one are "weakly conformal." Those which satisfy the earlier one automatically satisfy the one below.

(1') One or more compounds in each set may have an odd number of electrons, provided the number of odd-electron compounds in each set is the same and provided they fulfill $\left(3^{\prime}\right)$.

(2') Same as (2) in the definition of conformality.

$\left(3^{\prime}\right)$ Compounds which do not have LCAO eigenvalues of zero are stipulated to have an even number of electrons. If an odd-electron compound is present, it is stipulated to have a nondegenerate LCAO eigenvalue of zero. ${ }^{12}$

It is further stipulated that for the simplest LCAO results, where the dependence of Coulomb and exchange integrals on the over-all charge of an ion is ignored, each odd-electron compound in $\left(1^{\prime}\right)$ and $\left(3^{\prime}\right)$ can be replaced by its anion.

\section{DATA ON WEAK CONFORMALITY}

Examples of LCAO calculated $\pi$-electron energies ${ }^{11}$ of weakly conformal systems are given in Table III. The agreement of the two columns in the latter table is seen to be reasonably close $(0.1 \beta$ equals $2 \mathrm{kcal}$ mole ${ }^{-1}$ ), though not quite as good as that for strongly conformal systems. Examples are given in Table IV of $\pi$-electron energies of systems which simulate a semiquinone formation discussed later. Weak confor-

\footnotetext{
${ }^{12}$ For easy application, Property $\left(3^{\prime}\right)$ can be restated as follows, except for extremely rare cases for which a triply degenerate eigenvalue occurs. To eliminate such cases a condition which would be the analog of $(3 b)^{7}$ could presumably be added: ( $\left.3^{\prime} a\right)$ compounds which do not have LCAO eigenvalues of zero are stipulated to have an even number of electrons. If a compound has an odd electron then it is stipulated that one atom is left unconnected by the islands of polygons and that there is no way of connecting them without leaving this atom unconnected.
} 
TABLE III. LCAO $\pi$-electron energies of systems with radicals."

(16)

a Resonance energies are obtained by adding to $+E$ the number in parentheses at the beginning of each row.

mality requires not that $E^{(1)}$ equals $E^{(2)}$ but, as a later discussion shows, that $E^{(1)}-E^{(2)}$ be the same for all rows in the table.

Experimental data concerning weak conformality are presently limited. Several examples are available, however:

The set, benzyl radical plus butadiene, is weakly conformal to the set, allyl radical plus styrene. This conformality, not immediately apparent by superposition, is established in Appendix II. Experimental evidence for the additivity of their heats of combustion follows:

The sum of the heats of combustion of the first pair equals $\Delta H_{c}$ (toluene) $-D_{\text {benzyl-H }}-\Delta H_{c}(\mathrm{H})+\Delta H_{c}$ (butadiene), where $D$ is a dissociation energy and $\mathrm{H}$ a hydrogen atom. The sum of the heats of combustion of the second set equals $\Delta H_{c}$ (propylene) $-D_{\text {allyl-H }}-$ $\Delta H_{c}(\mathrm{H})+\Delta H_{c}$ (styrene). Now $\Delta H_{c}$ (styrene) $+\Delta H_{c}$ (butadiene) is $1552.1 \mathrm{kcal} \mathrm{mole}^{-1}$ and $\Delta H_{\mathrm{c}}$ (propylene) $+\Delta H_{c}$ (styrene) is $1552.0 \mathrm{kcal} \mathrm{mole}{ }^{-1} .10$ Thus, the weak conformality of the sets implies that $D_{\text {benzyl-H }}$ should equal $D_{\text {allyl-H, }}$ though perhaps not as closely as it would be if the relevant systems had been strongly conformal. The actual dissociation energies are about 83 and $80 \mathrm{kcal} \mathrm{mole}^{-1}$, respectively, ${ }^{13}$ which is quite close agreement. Typically, the difference of dissociation energies $D_{\text {benzyl- } y}$ and $D_{\text {allyl-y }}$ is about $2 \mathrm{kcal}$ mole ${ }^{-1}$ for various $y^{\prime}$ s. ${ }^{13}$

Another example of weak conformality involves semiquinone formation constants. (Diagrams are given in

${ }^{13}$ See, for example, survey in A. Streitweiser, Jr., Molecular Orbital Theory (John Wiley \& Sons, Inc., New York, 1961).
Table IV, with $\mathrm{C}$ replaced by an oxygen atom and with OH's presumed not to take part in the conjugation in the case of the LCAO model.) If all components in the semiquinone formation,

$$
\mathrm{QH}_{2}+\mathrm{Q}=2 \mathrm{QH}
$$

bear the same charge then one would expect the entropy of reaction to be not only small but to largely cancel for different Q's. By weak conformality the heat of reaction should be independent of $Q$. (The latter situation might also prevail if each $\mathrm{OH}$ were conjugated in $\mathrm{QH}$ and $\mathrm{QH}_{2}$ to the same extent.) Thus, the free energy of the above reaction should be independent of $Q$. It has been reported from measurements of redox potentials as a function of $p \mathrm{H}$ that this $\Delta F^{o}$ is independent of Q.14 However, for most of the available examples it appears that the $\mathrm{H}$ 's in $\mathrm{QH}_{2}$ are not equivalent, one often being attached to an $\mathrm{N}$ and the other to an $\mathrm{O}$. The arguments given above apply only to the case of equivalent hydrogens, and further measurements on recent systems of this nature ${ }^{15}$ would be desirable.

Another example involves dissociation energies of phenolic compounds $D_{\mathrm{RO}-\mathrm{H}}$. The third diagram in Table III for an LCAO model suggests that if the $\mathrm{OH}$ does not conjugate with the ring then according to weak conformality the dissociation energy should be independent of $R$, again perhaps not with the same accuracy expected for strongly conformal behavior. The data are not known accurately, but Hush $^{16}$ has made an analysis of some electrochemical data of Fieser. His assumptions then led to the following estimates for $D_{\text {RO-H. }}$. When RO-H was phenol, 2-phenanthrol, $p$-hydroxydiphenyl, 2-naphthol, 1-naphthol, and

TABLE IV. LCAO $\pi$-electron energy differences for simulated semiquinone formation.

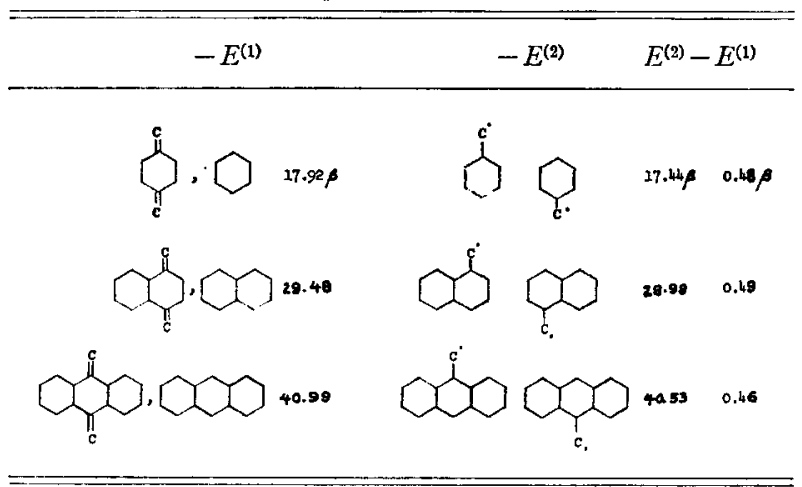

${ }_{14} \mathrm{G}$. Schwarzenbach, Inst. Intern. Chim. Solvay, Conseil Chim. 1950,47 , remarks briefly on this point.

${ }_{15}$ For example, the equilibrium constant for Reaction (1) when $Q$ is duroquinone is obtained by combining the data of $I$. Yamazaki and L. H. Piette, J. Am. Chem. Soc. 87, 986 (1964), with that of J. H. Baxendale and co-workers, Trans. Faraday Soc. 49, 1140, 1433 (1953).

${ }^{16}$ N. S. Hush, J. Chem. Soc. 1953, 2375. 
1 -anthrol, $D_{\text {Ro-H }}$ was roughly $82-84,81,81,80,75$, and $71 \mathrm{kcal} \mathrm{mole}^{-1}$, respectively. Except for the last two values, which may reflect inductive or overcrowding effects, these results are fairly close in spite of their approximate nature.

\section{NEARLY CONFORMAL SETS}

"Nearly conformal" sets have exactly the same number of two-step random walks (a condition ensured by having the same number of conjugated bonds) and almost the same number of four-step random walks (as well as of longer ones).

One example is the set styrene +propylene, which is nearly conformal to toluene+butadiene, as one may see by superposition (Appendix II) or from calculations based on Table V. The sum of the heats of combustion of the first set is 1552.0 while that of the second is $1552.0 \mathrm{kcal}$ mole ${ }^{-1} .^{10}$ The LCAO $\pi$-electron energies are -12.42 and $-12.47 \beta$, respectively. ${ }^{11}$ For later use in Eq. (16) we note that the LCAO polynomials, $\Delta(x)$, are:

styrene, propylene, $\quad x^{10}-9 x^{8}+27 x^{6}-35 x^{4}+20 x^{2}-4$; toluene, butadiene, $\quad x^{10}-9 x^{8}+28 x^{6}-37 x^{4}+21 x^{2}-4$.

Other nearly conformal sets can be found in the linear, branched, and exocyclic polyenes considered by Dewar and Gleicher, ${ }^{1}$ and both LCAO and SCFLCAO-MO additivity of energies was found for them.

\section{RANDOM WALKS AND NEAREST-NEIGHBOR DEPENDENT BONDS}

In Appendix III it is shown that the numbers of two-, four-, and six-step self-returning walks in a molecule can be expressed in terms of the numbers of nearest-neighbor dependent bonds and of different rings. The final results are given in Table $V$. It is seen that sets which have similar numbers of corresponding bonds $\left(\pi^{3}-\pi^{3}, \pi^{3}-\pi^{2}, \pi^{2}-\pi^{2}\right)$ and rings are conformal up to and including six-step walks.

By way of example, this result can be used to show that the "bond-energy additivity" of a recent study"

TABLE V. Numbers of walks assigned to each bond type.

\begin{tabular}{ccccc}
\hline Bond & Two-step & Four-step & Five-step & Six-step \\
\hline$\pi-\pi$ & 2 & 2 & 0 & 2 \\
$\pi-\pi^{2}$ & 2 & 4 & 0 & 8 \\
$\pi-\pi^{3}$ & 2 & 6 & 0 & 18 \\
$\pi^{2}-\pi^{2}$ & 2 & 6 & 0 & 20 \\
$\pi^{2}-\pi^{3}$ & 2 & 8 & 0 & 36 \\
$\pi^{3}-\pi^{3}$ & 2 & 10 & 0 & 58 \\
$\cdots$ & & & & \\
Extra for six ring & 0 & 0 & 0 & 12 \\
Extra for five ring & 0 & 0 & 10 & 0 \\
\hline
\end{tabular}

TABLE VI. Numbers of nearest-neighbor dependent bonds and of rings in fused aromatics.

\begin{tabular}{lcccc}
\hline \multicolumn{1}{c}{ Compound } & $\pi^{3}-\pi^{3}$ & $\pi^{3}-\pi^{2}$ & $\pi^{2}-\pi^{2}$ & Rings \\
\hline Benzene & 0 & 0 & 6 & 1 \\
Naphthalene & 1 & 4 & 6 & 2 \\
Anthracene & 2 & 8 & 6 & 3 \\
Phenanthrene & 3 & 6 & 7 & 3 \\
Naphthacene & 3 & 12 & 6 & 4 \\
Benz(a) anthracene & 4 & 10 & 7 & 4 \\
Chrysene & 5 & 8 & 8 & 4 \\
Triphenylene & 6 & 6 & 9 & 4 \\
\hline
\end{tabular}

can be regarded as a special case of six-step conformalset additivity. We consider first the fused-ring aromatics which appeared there. They are listed in Table VI. From that table or by simple geometrical superposition one sees that the following sets are conformal up to six steps: anthracene+benzene $\rightarrow$ two naphthalenes; naphthacene+benzene $\rightarrow$ anthracene + naphthalene; benz(a) anthracene+benzene $\leftrightarrow$ phenanthrene+ naphthalene. From Tables V and VI (but not obvious by superposition) six-step conformality also exists for: chrysene + naphthacene $\leftrightarrow$ two benz (a) anthracenes; triphenylene+anthracene $\leftrightarrow$ phenanthrene+chrysene.

Thus, the only conformally independent compounds are benzene, naphthalene, and phenanthrene, or any three compounds derived from them by conformal arguments. Conformality then implies that the heats of combustion (and MO resonance energies) of all remaining compounds are linearly expressible in terms of any such three. One can do this in various ways, for example by assigning "energies" to $\pi^{2}-\pi^{2}, \pi^{2}-\pi^{3}$, and $\pi^{3}-\pi^{3}$ bonds. A striking additivity, such as that observed by McGinn, ${ }^{2}$ then results. McGinn also treated several other conjugated compounds but of the nonfused ring variety. They are discussed in Appendix IV.

\section{RELATION OF SECULAR DETERMINANTS TO RANDOM WALKS}

In the present section a relation is derived between the number and quality of self-returning random walks and the coefficients of a polynomial arising from the LCAO secular determinant. The primary purpose of the derivation is to interpret in the next section the additivity of LCAO $\pi$-electron energies described earlier. The energies in Tables II to IV were computed by making all Coulomb integrals $\alpha_{i}$ equal, all exchange integrals $\beta_{i j}$ equal for adjacent $i$ and $j$, and all overlap integrals $S_{i j}$ zero for $i \neq j$. In the proof, this approximation is therefore later introduced. However, at first we distinguish between the various $\alpha_{i}$ and between the $\beta_{i j}$, partly to give some LCAO notion as to quality of a random walk. All $S_{i j}$ are set equal to zero for $i \neq j$. 
The difference between $\alpha_{i}$ and the $\alpha$ of some "standard" carbon atom $\alpha_{\mathrm{C}}$ is denoted by $\gamma_{i}$. The eigenvalues $\epsilon_{k}$ measured relative to $\alpha_{\mathrm{C}}$ are the roots of the secular equation (2).

$$
\Delta(\lambda)=|\mathbf{B}-\lambda \mathbf{I}|=0,
$$

where $B$ is a symmetric matrix whose elements $b_{i j}$ are

$$
b_{i j}=\beta_{i j}+\gamma_{i} \delta_{i j},
$$

where $\delta_{i j}$ is the Kronecker delta. The $\beta_{i j}$ vanish when $i=j$. The determinant can be written in powers of $\lambda$,

$$
\Delta(\lambda)=\sum_{m=0}^{m=n} b_{m} \lambda^{n-m}
$$

where $n$ is the number of rows (columns) in the determinant and the $b_{m}$ depend ${ }^{17}$ on the traces $s_{k}$ of various powers $\mathbf{B}^{k}$ of the matrix $\mathbf{B} ; n$ is the number of atoms in the conjugated system;

$$
\begin{aligned}
s_{k} & =\operatorname{Tr} B^{k}, \\
-m b_{m} & =\sum_{i=1}^{m} s_{i} b_{m-i}, \\
b_{0} & =1 .
\end{aligned}
$$

We have

$$
\begin{aligned}
\operatorname{Tr} \mathbf{B} & =\sum_{i=1}^{n} b_{i i}, \\
\operatorname{Tr} \mathbf{B}^{2} & =\sum_{i, j=1}^{n} b_{i j} b_{j i}, \\
\operatorname{Tr} \mathbf{B}^{3} & =\sum_{i, j, k=1}^{n} b_{i j} b_{j k} b_{k i}, \text { etc. }
\end{aligned}
$$

Thus, each trace can be the sum of many terms, each of which begins and ends on some Atom $i$. Parenthetically, we note that when all $\gamma_{i}$ are made to vanish each term in any trace is a self-returning random walk from atom to atom, in which each step $i j$ is assigned a weight $b_{i j}$ equal to $\beta_{i j}$.

If all $\gamma_{i}$ were set equal to zero and all $\beta_{i j}$ equal to 1 for adjacent $i$ and $j$, then $\lambda$ would be in units of $\beta$ and $s_{k}$ would equal the number of self-returning random walks of $k$ steps. One then finds that $-b_{1},-2 b_{2},-3 b_{3}$, and $-4 b_{4}$ equal $0, s_{2}, s_{3}$, and $s_{4}-\frac{1}{2} s_{2}{ }^{2}$, respectively, and so on. However, we do not introduce these approximations for the $\gamma_{i}$ and $\beta_{i j}$ as yet.

Two compounds, (1) and (2), are assigned properties denoted by subscripts or superscripts (1) and (2). The product of their secular determinants, $\Delta_{(1)} \Delta_{(2)}$, is

${ }_{17}$ E. Bodewig, Matrix Calculus (North-Holland Publishing Company, Amsterdam, 1959), p. 395. A result analogous to (6) has been obtained in a less direct way by $\mathrm{Hs}$. $\mathrm{H}$. Gunthard and $\mathrm{H}$. Primas, Helv. Chim. Acta 39, 1645 (1956). However, their result differs from (6) for $m \geq 6$ and (unless this writer misunderstands their symbols) yields incorrect results for well-known LCAO polynomials. Many useful LCAO polynomials are given in Ref. 18.

${ }_{18}$ Polynomials are taken from those of the individual compounds given by E. Heilbronner, Helv. Chim. Acta 36, 170 (1953). denoted by $\Delta_{\mathrm{I}}$ and can be written as

where

$$
\Delta_{\mathbf{I}}(\lambda)=\left|\mathbf{B}_{\mathbf{I}}-\lambda \mathbf{I}\right|
$$

$$
\mathbf{B}_{\mathrm{I}}=\left(\begin{array}{cc}
\mathbf{B}_{(1)} & \mathbf{0} \\
\mathbf{0} & \mathbf{B}_{(2)}
\end{array}\right) .
$$

From this expression it follows that

i.e.,

$$
\operatorname{Tr} B_{I}^{m}=\operatorname{Tr} B_{(1)}^{m}+\operatorname{Tr} B_{(2)}^{m},
$$

$$
s_{m}{ }^{\mathrm{I}}=s_{m}{ }^{(1)}+s_{m}{ }^{(2)} \text {. }
$$

The $s_{m}{ }^{\mathrm{I}}$ are related to the coefficients $b_{l k}^{\mathrm{I}}$ of the $\lambda^{n-k} \mathrm{~s}$ in $\Delta_{\mathrm{I}}(\lambda)$ according to Eq. (6).

The determinants $\Delta_{\mathrm{I}}$ and $\Delta_{\mathrm{II}}$ of the two conformal sets may now be compared. Coefficients of corresponding powers of $\lambda^{k}$ will be equal if all corresponding $s_{i}$ are equal for $i$ less than or equal to $k$. If to each walk in Set $I$ (including silent counts ${ }^{19}$ ) one can assign a walk of the same quality in Set II, and conversely, up to $m$ (audible ${ }^{19}$ ) steps, then $s_{m}{ }^{I}$ and $s_{m}{ }^{\text {II }}$ are equal, as are corresponding coefficients of $\lambda^{k}$ for $k$ greater than or equal to $n-k$.

Even when coefficients of powers of $\lambda$ less than $n-m$ are not exactly equal, because of a nonexact matching of walks of more than $m$ steps, they are found to be approximately equal, perhaps partly because many of these longer walks are still local in nature; they involve much retracing of steps. In the conformal sets in Fig. 3 the walks in one set which have no match in those of the other are walks going from an $R$ to an $S$ group and back. It would be very desirable to prove that the coefficients of low powers of $\lambda$ should be fairly close. We have merely hinted at a possible reason.

As an example of the similarity of the secular determinants of conformal sets we consider stilbene tethylene and two styrenes. For reasons described in the next section we replace $\lambda$ by $i y$ :

stilbene+ethylene, ${ }^{18}$

$$
\begin{aligned}
\Delta(i y)=y^{16}+16 y^{14}+102 y^{12}+337 y^{10} & +633 y^{8}+694 y^{6} \\
& +432 y^{4}+137 y^{2}+16 ;
\end{aligned}
$$

two styrenes, ${ }^{18}$

$$
\begin{aligned}
\Delta(i y)=y^{16}+16 y^{14}+102 y^{12}+336 y^{10} & +625 y^{8}+672 y^{6} \\
& +408 y^{4}+128 y^{2}+16 .
\end{aligned}
$$

19 The reason for retaining this "quality" is based on the feeling by the writer that LCAO additivity will persist if the differences in quality are not too large. If the $\gamma_{i}$ are not made to vanish, each term in the trace still represents a self-returning random walk, but one in which one marks time silently for one "step" on the $j$ th atom whenever $b_{j j}$ appears. Each self-returning random walk has a certain quality described by the sequence of $b_{i j}$ 's appearing in it. Two walks in two different compounds have the same "quality" if the sequence of $b_{i j}$ 's in each correspond, i.e., if they pass through a sequence of atoms, corresponding ones of which are chemically similar. The two-step and four-step random walks employed in the definition of conformality do not count marking time on a particular atom as a step in the two or the four steps. Rather, it is included as a silent count. Silent counts help determine the quality of a walk. 
The difference in coefficients of $y^{10}$ is due to a difference of six-step walks. By contrast, the following sets are not conformal:

1,2-dimethylene cyclohexadiene+benzene,

$$
-\Delta(i y)=y^{14}+14 y^{12}+75 y^{10}+197 y^{8}+273 y^{6}
$$

$$
+195 y^{4}+61 y^{2}+4
$$

two benzyls,

$$
-\Delta(i y)=y^{14}+14 y^{12}+75 y^{10}+196 y^{8}+267 y^{6}
$$

$$
+182 y^{4}+49 y^{2} \text {. }
$$

These sets are geometrically superposable (and would otherwise be four-step conformal) but do not satisfy Condition (3). The $\Delta$ 's differ primarily in the coefficient of $x^{0}$. The resulting difference in LCAO energies is ${ }^{11} 0.51 \beta$; that of the preceding (conformal) pair of sets is only $0.03 \beta$. We return to this large difference in the next section.

\section{RELATION OF LCAO CHARACTERISTIC POLYNOMIAL TO $\pi$-ELECTRON ENERGY}

The formula used to calculate the ground-state $\pi$ electron energies $E$ in Table II is equivalent to the following ${ }^{20}$ :

$$
E=\frac{1}{\pi i} \oint_{\Gamma} z \frac{d}{d z} \log \Delta(z) d z
$$

where $z$ is a complex variable, $\Gamma$ is a contour which encloses the doubly occupied orbitals, and $\Delta(z)$ is the LCAO secular determinant with the usual $\lambda$ replaced by $z$. [The formula as given in (15) is for an even number of electrons.] When the energy of each occupied orbital of each compound in both sets is negative, as is the case for the molecules in Table II, the same contour $\Gamma$ can be chosen for all compounds.

Equation (15) is normally applied to individual compounds. However, there is no objection to applying it to a set of compounds if their occupied orbitals lie inside the same $\Gamma$. In that case $E$ is the sum of their $\pi$-electron energies and $\Delta(z)$ is the product of their secular determinants. The difference of $\pi$-electron energies $E_{\mathrm{I}}-E_{\mathrm{II}}$ of the two such sets (i.e., sets having the same $\Gamma$ ) can be written as in (15) with $\Delta(z)$ replaced by $\Delta_{\mathrm{I}}(z) / \Delta_{\mathrm{II}}(z)$. One may take $\Gamma$ to be a contour along the imaginary axis and around a large semicircle in the negative half of the complex plane. The value of the integral on the semicircle vanishes in the expression for $E_{\mathrm{I}}-E_{\mathrm{II}}$ when the radius of the semicircle tends to infinity. Upon subsequent integra-

${ }^{30}$ C. A. Coulson, Proc. Cambridge Phil. Soc. 36, 201 (1940); J. Chem. Soc. 1954, 3111. tion by parts along the imaginary axis one obtains (16):

$$
E_{\mathrm{I}}-E_{\mathrm{II}}=-\frac{1}{\pi} \int_{-\infty}^{\infty} \log \frac{\Delta_{\mathrm{I}}(i y)}{\Delta_{\mathrm{II}}(i y)} d y
$$

For subsequent discussion it is convenient to divide the integration interval in (16) into two parts: (a) $|y|>1$ and (b) $|y|<1$. For typical systems, the major contribution to each $\log \Delta(i y)$ in Region (a) comes from the high powers of $y$ and that in Region (b) comes from low powers.

The $\pi$-electron energies are seen to be equal when their secular determinants are equal. We saw that the coefficients of the high powers of $y$ in $\Delta_{\mathrm{I}}(i y)$ and $\Delta_{\mathrm{II}}(y)$ are exactly equal and noted (but did not prove) that the other coefficients are fairly close. Thus, the value of the integral in Region (a) should be small and, unless one $\Delta(i y)$ has a factor proportional to $y$, one would expect the value of the integral in Region (b) to be small also. When a compound in Conformal Set $I$ has an eigenvalue of zero, $\Delta_{\mathrm{I}}(i y)$ has a factor $y$ and the integrand then has a logarithmic singularity at the origin. Although the integrand is still integrable, the value of the integral in Region (b) will now be large, as in the nonconformal set mentioned in the preceding section. For this reason, Condition (3) was imposed. It eliminates zero roots: Inspection of the expansion of $\Delta(\lambda)$ shows that the constant term vanishes unless Conditions ${ }^{7}(3 a)$ and (3b) are fulfilled.

Suppose now that one compound in Set $I$ and one in Set II each has a nondegenerate root at $\lambda=0$. Then, each of $\Delta_{\mathbf{I}}(i y)$ and $\Delta_{\mathrm{II}}(i y)$ has a factor $y$ which cancels in the ratio $\Delta_{\mathrm{I}}(i y) / \Delta_{\mathrm{II}}(i y)$. Thus, the integrand once again has the desirable behavior of not having a major contribution near $y=0$, and one would expect the $\pi$-electron energies $E_{\mathrm{I}}$ and $E_{\mathrm{II}}$ to again be essentially equal. Accordingly, weak conformality was introduced so that an equal number of compounds in each conformal set can have a nondegenerate root at $\lambda=0$. If there are more than two compounds per set, the condition $\left(3^{\prime}\right)$ can still be fulfilled, the main aim being to effect a cancellation of all $y$ factors in the product $\Delta_{\mathrm{I}}(i y) / \Delta_{\mathrm{II}}(i y)$. For this reason ratios of equilibrium constants of semiquinones would be expected to be equal since the comparison is of compounds which fulfill this $\left(3^{\prime}\right)$ :

$$
\mathrm{Q}^{\mathrm{a}} \mathrm{H}_{2}, \mathrm{Q}^{\mathrm{a}}, 2 \mathrm{QH}^{\mathrm{b}} \leftrightarrow \mathrm{Q}^{\mathrm{b}} \mathrm{H}_{2}, \mathrm{Q}^{\mathrm{b}}, 2 \mathrm{QH}^{\mathrm{a}},
$$

where $Q^{a}$ and $Q^{b}$ denote different quinones.

In writing (15) we have restricted attention to doubly occupied orbitals, i.e., to compounds with even number of electrons. However, any root at $z=0$ does not contribute to (15), and thus it does not matter whether, from the viewpoint of LCAO calculations, there are one or two electrons in this highest occupied level. Thus, in the case of the modified condition $\left(3^{\prime}\right)$ the condition (1) that the number of electrons be even was removed for such compounds with an LCAO eigenvalue of zero. 
Bet I

(a)

$$
\begin{gathered}
23456 \\
c=c-c=c-c=c \\
c=c \\
314
\end{gathered}
$$

(b)<smiles></smiles>

\section{BOND ORDERS, FREE VALENCE, AND CHARGE DENSITIES}

The LCAO expression for the bond order $p_{r s}$ of the $r s$ bond can be expressed as an integral ${ }^{21}$

$$
p_{r s}=-\frac{1}{2 \pi} \int_{-\infty}^{\infty} \frac{\partial}{\partial \beta_{r s}} \log \Delta(i y) d y,
$$

when all the roots lie in $\Gamma$ as discussed in the previous section. [If some of these roots in $\Gamma$ were positive but less than $a$, one would have $\Delta(a+i y)$ instead of $\Delta(i y)$. In (18) $\beta_{r s}$ is the exchange integral for the $r s$ bond.

We now superpose the compounds in one of the sets and assign to the line joining the $r$ th and $s$ th sites the same label $r s$ (and $\beta$ is labeled $\beta_{r s}$ ) in each compound. Similar assignments are then made to the corresponding line in two superposed compounds of the second set. If $\Delta(i y)$ in (18) is the product of the determinants of the two compounds in one set, then (18) is the sum of their LCAO bond orders for this $r$ th bond. The coefficients of any power of $y$ in $\Delta(i y)$ involve sums of various products of the $\beta_{i j}$ 's, it is recalled. If there is established a 1:1 correspondence between walks in the two sets and if the quality of corresponding walks

${ }^{21}$ C. A. Coulson and H. C. Longuet-Higgins, Proc. Roy. Soc. (London) A191, 39 (1947).
Eet II wo butedienes

$$
\begin{aligned}
& 1234 \\
& \mathrm{c}=\mathrm{c}-\mathrm{c}=\mathrm{C} \\
& \mathrm{c}=\mathrm{c}-\mathrm{c}=\mathrm{c} \\
& \text { 3.4156 }
\end{aligned}
$$

Fig. 1. Conformal sets: Hexatriene+ethylene is four step conformal with two butadienes. Anthracene + benzene is six step conformal with two naphthalenes. The numbering is also used to identify bonds in the text. is similar, then $\beta_{r s}$ enters $\Delta_{\mathbf{I}}(i y)$ in the same way it enters $\Delta_{\mathrm{II}}(i y)$, for the appropriate powers of $y$, and then the sum of the bond orders at each site $r s$ of one set equals the corresponding sum in the other set.

LCAO results ${ }^{11}$ for several compounds are given below. The numbering for the first two sets is given in Fig. 1. The numbering for the third and fourth sets is given in Fig. 2. The following numerical values are to be divided by 100 to obtain the bond order: For anthracene+benzene the average LCAO order of the $1-2,2-7,2-3,3-4$ and $4-5$ bonds is $64,58,54,74$, and 59 , respectively, while for the two naphthalenes it is $64,56,56,72$, and 60 . For hexadiene+ethylene the $1-2,3-4$, and $3-4$, average bond orders are 87,48 , and 90 , while for two butadienes they are 89,45 , and 89. For stilbene+ethylene the $7-8,8-9,9-10$, 10-11, and 11-12 average bond orders are 91, 43, 60, 68 , and 66 , respectively, while for two styrenes they are $91,41,61,68$, and 66 . For dibenzofulvene +fulvene the $1-14,1-2,2-3,3-4,4-5,5-6,6-7,7-8$, and $2-7$ average bond orders are $79,42,62,67,65,67,62,46$, and 67. For two benzofulvenes they are 80, 42, 63, 67, $65,68,60,45$, and 68 . The largest discrepancy in the sum of bond orders between the two sets for any bond is seen to be about 0.02 per compound. A difference of 0.02 in bond order corresponds roughly to a difference of $0.005 \AA$ in predicted bond length. 
Set I

(a)

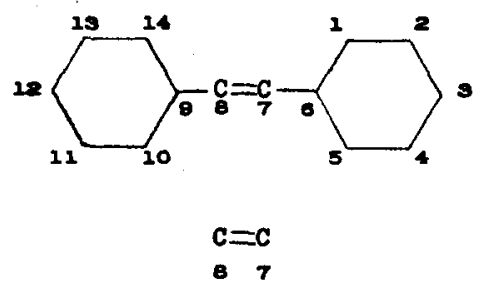

Fig. 2. Numbering of atoms Tin conformal sets used to identify bonds in the text. (b)
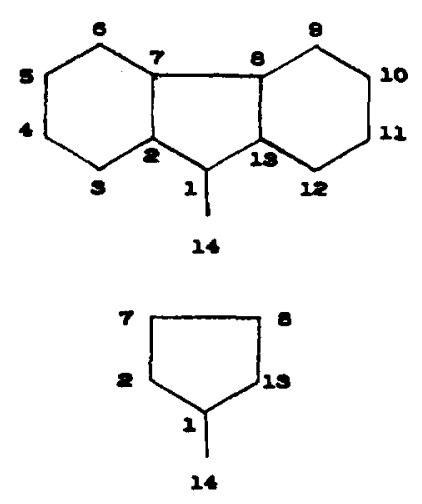

Bet II

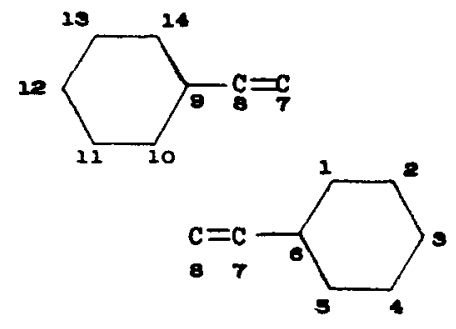

Coulson has defined for MO Theory the "free valence" of an atom $r$ as equal to some constant minus the sum of the bond orders of bonds terminating on $r^{22}$ This quantity has been employed in some discussions of reactivity. Thus, the sum of the LCAO free valences of the two compounds at a site $r$ in one set equals the corresponding sum for the second set.

For anthracene +benzene the average bond length of the $1-2,2-7,2-3,3-4$, and $4-5$ bonds are $1.39_{5}, 1.41_{5}$, $1.42_{5}, 1.37,1.41$ and for two naphthalenes they are $1.39_{5}, 1.39_{5}, 1.42_{5}, 1.36_{5}, 1.40_{5}$, respectively. ${ }^{13}$ The numbering is that given in Fig. 1 .

A formula analogous to (18) but involving differentiation with respect to a Coulombic integral $\alpha_{r}$ has been derived for charge densities. ${ }^{21}$ In LCAO calculations of alternant hydrocarbons having all $\alpha_{r}$ equal these charge densities at each conjugated atom are equal. When the $\alpha_{r}$ 's are not all equal an LCAO additivity would be expected if corresponding walks have similar quality, but we have not examined the LCAO results.

\section{ACKNOWLEDGMENTS}

The writer is most indebted to Miss Mary Attermeyer for her assistance in the compilation of Table I

${ }^{22}$ C. A. Coulson, Trans. Faraday Soc. 42, 106, 265 (1946). in this paper and for helpful discussions. He greatly appreciates a number of very helpful suggestions made by Professor E. M. Loebl.

\section{APPENDIX I. CONFORMALITY AND SUPERPOSITION}

Fulfilment of Conformality Conditions (2) can often be recognized by proper superposition of the two compounds in one set and comparing with similarly superposed compounds in a second set. Two examples are given below, the first involving four-step and the second involving six-step conformality. In the superposition of atoms of each compound in a set certain regions in one set can be made to correspond in a one-to-one manner with regions of a second superposed set in such a way that there is an exact matching in number and quality of the self-returning random walks that begin in corresponding regions.

In Fig. 1(a) Set I consisting of hexatriene and ethylene conforms to Set II consisting of two butadienes. Similarly numbered atoms within a set are to be superposed. Primes are used to distinguish these atoms for purposes of describing random walks. The regions (12), (56), and $\left(3,4,3^{\prime}, 4^{\prime}\right)$ in Set I of Fig. 1(a) correspond to Regions (12), (56), and $\left(3,4,3^{\prime}, 4\right)$ in Set II, respectively. An example of a four-step returning walk 


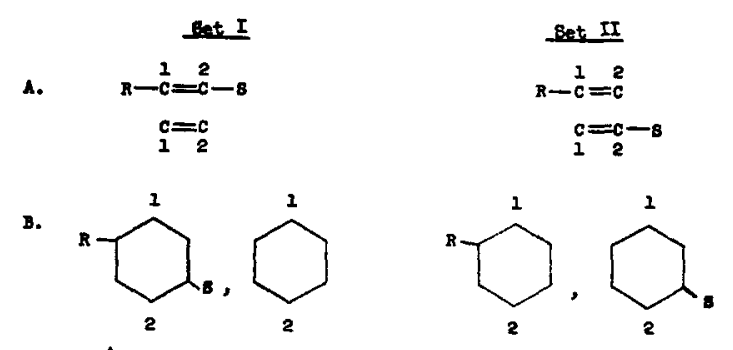

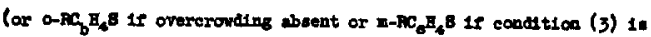
forteriled.)

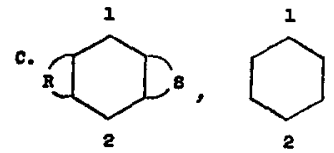<smiles>C1CCC2CCCC2C1</smiles><smiles>CC1CC2CC2CC2CCCC12</smiles><smiles>CC1CCCCC1</smiles><smiles>CC1CCCC2CNCC2C1</smiles><smiles>CC1CC2CCC2C1</smiles>

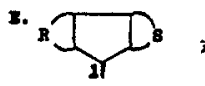<smiles>CC1CCCCC1</smiles><smiles>[C]1CCC2CCC12</smiles><smiles>[C]1CCC2CCC12</smiles>

F.
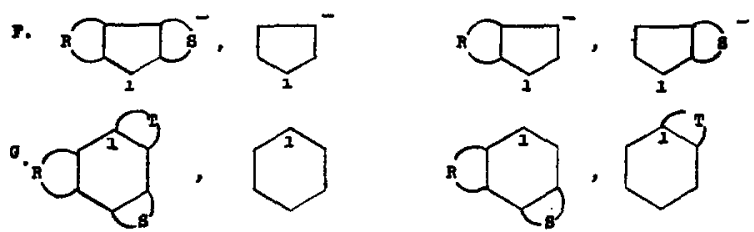

FIG. 3. Examples of conformal sets, where $\mathbf{R}$ and $\mathbf{S}$ denote conjugated, partially conjugated or nonconjugated groups, provided Condition (3) of conformality is fulfilled.
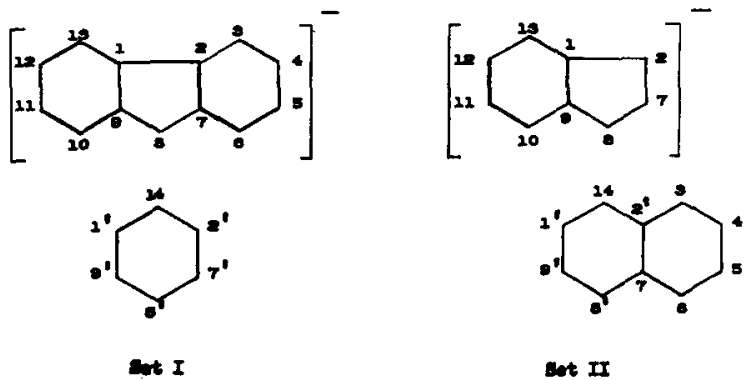

sot II

FIG. 4. An example of four-step conformality (cf. Appendix I).

is $(3,4,5,4,3)$ in Set $I$, which corresponds to $\left(3^{\prime}, 4^{\prime}\right.$, $\left.5,4^{\prime}, 3^{\prime}\right)$ in Set II.

The set anthracene+benzene conforms to a set consisting of two naphthalenes [Fig. 1(b)]. The regions $\left(9,10,11,12,13,14,9^{\prime}, 14^{\prime}\right),\left(2,3,4,5,6,7,2^{\prime}, 7^{\prime}\right)$, $\left(1,1^{\prime}\right)$, and $\left(8,8^{\prime}\right)$ in the first set correspond to $(9,10$, $\left.11,12,13,14,9^{\prime}, 14^{\prime}\right),\left(2,3,4,5,6,7,2^{\prime}, 7^{\prime}\right),\left(1,1^{\prime}\right)$ and $\left(8,8^{\prime}\right)$ in the second set.

Some classes of conformal sets are listed in Fig. 3, where similarly numbered atoms in a set are to be superimposed. $\mathbf{R}$ and $\mathbf{S}$ denote conjugated or nonconjugated systems. The sets conform only as long as overcrowding of molecules is absent or occurs to the same extent in the two sets. For example, overcrowding might occur in Set I of Classes $D$ and $G$. Sets in Class $B$ (if the substitution is $p$ or $m$ ) and in $C$ are six-step conformal. The remainder are four-step conformal.

The four-step conformality in Class $F$ can be seen as follows. In Fig. 4 the regions $(1,9,10,11,12,13$, $14)^{*},\left(1^{\prime}, 9^{\prime}\right),(3,4,5,6)^{*},\left(2,2^{\prime}, 7,7^{\prime}\right)^{*}$, and $\left(8,8^{\prime}\right)$ in Set $I$ correspond to $(1,9,10,11,12,13,14)^{*}$, $\left(1^{\prime}, 9^{\prime}\right),(3,4,5,6)^{*},\left(2,2^{\prime}, 7,7^{\prime}\right)^{*}$, and $\left(8,8^{\prime}\right)$ in Set II, respectively. Some of the walks in the asterisked regions may differ in quality in the actual molecules, though not in simple LCAO calculations where all atoms are made similar. For example, Walk $(2,1,2$, $3,2)$ in I is matched to $\left(2^{\prime}, 14,2^{\prime}, 3,2^{\prime}\right)$ in II.

Azulene and naphthalene are four step conformal in the simplest LCAO case (all $\alpha_{i}$ 's and $\beta_{i j}$ 's equal). While not obvious from geometrical superposition one can see this conformality as follows: There is a matching of numbers of random walks in these two molecules for walks confined to the perimeter, for there are 10 atoms on the perimeter of each compound. However, any two-step and four-step random walk in azulene in which at least one step is along the crosslink also has its immediate counterpart in a similar walk involving the crosslink in naphthalene. The matching of numbers of walks therefore follows, but the dissimilarity in quality is perhaps reflected in the large difference in heats of combustion ( 1279 vs $1250 \mathrm{kcal} \mathrm{mole}^{-1}$ ). The simple LCAO $\pi$-electron energies are essentially equal $(-13.64$ and $-13.68 \beta)$.

\section{APPENDIX II. WEAK AND NEAR CONFORMALITY AND SUPERPOSITION}

The set, benzyl radical plus butadiene, is weakly conformal to the set, allyl radical plus styrene. That these sets have similar numbers of four-step as well as of two-step random walks is immediately evident from a comparison of the coefficients of the first three terms in the products of the LCAO polynomials of these sets. (The relation of the latter to the former is discussed in the text). However, the conformality can also be recognized as follows from Fig. 5, though less
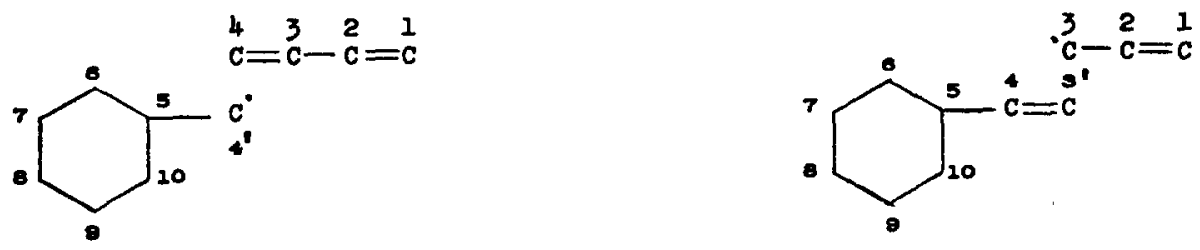

Fic. 5. Nearly conformal sets (cf. Appendix II). 
obviously than the conformality in Figs. 1 and 2 : Regions $(6,7,8,9,10),\left(2,3,4,4^{\prime}, 5\right)$, and (1) in Set I correspond to Regions $(6,7,8,9,10),\left(2,3,3^{\prime}\right.$, 4,5 ), and (1) in Set II, respectively. All four-step walks involving sequences on Atoms $(2,3,4)$ in Set I are matched in numbers at least by all four-step walks involving sequences on Atoms $\left(3^{\prime}, 4,5\right)$ in Set II. Matching of all other four-step walks is as straightforward as it was in the earlier examples. For present purposes of prediction we assume that the quality of all corresponding walks is similar, or that differences in quality are "averaged out."

The set styrene+propylene is nearly conformal to toluene + butadiene:

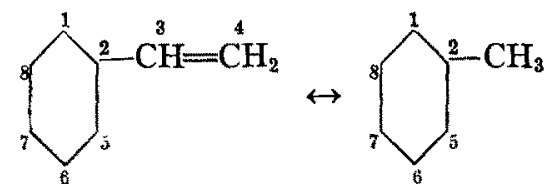<smiles>C=CC</smiles><smiles>C=CC=C</smiles>

Similarly numbered atoms are to be superimposed. The $\mathrm{CH}_{3}$ group in one set roughly cancels that in the other. The quality of some corresponding walks may not be as close as that in conformal sets. [Compare the walk $(2,1,2,3,2)$ on the left with its corresponding four-step walk $\left(2^{\prime}, 1^{\prime}, 2^{\prime}, 3,2^{\prime}\right)$ on the right.] Most four-step random walks on the left have corresponding walks on the right, but the four walks involving Atoms 5,2 , and 3 , simultaneously, do not.

\section{APPENDIX III. RANDOM WALKS AND NEAREST-NEIGHBOR DEPENDENT BONDS}

We first enumerate the all types of two-, four-, and six-step walks involving Bond Atoms A and B simultaneously. Some of them involve nearest neighbors in addition.

For two-step walks there are only two distinguishable walks (ABA, BAB).

For four-step walks: (a) There are four such walks involving three atoms simultaneously, as seen from Fig. 6(c) (CABAC, ACABA, ABACA, BACAB). (b) There are two walks involving only two atoms $A$ and B simultaneously.

For six-step walks: (a) There are six such walks involving the four atoms in Fig. 6(a) simultaneously. (b) There are 12 walks involving the four atoms in Fig. 6(b) simultaneously. (c) There are 12 walks involving the three atoms in Figure 6(c) simultaneously. (d) There are two walks involving the two atoms alone [Fig. 6(d)].

Certain of these walks can be uniquely assigned to the $\mathrm{AB}$ bond while only a certain fraction of the others

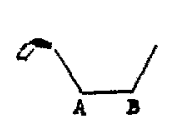

(a)

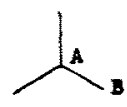

(b)

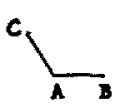

(c)

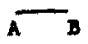

(d)
Frg. 6. Illustration used to count numbers of self-returning random walks for various nearest-neighbor dependent bonds.

can be assigned to Bond $\mathrm{AB}$ if double counting is to be avoided in the count of total numbers of two-step, four-step, and six-step walks in the molecule. With this restriction in mind one finds: the Type (b) four-step walk can be assigned to the $A B$ but only one-half of the Type (a) four-step walks can be so assigned. The Type (a) and (d) six-step walks can be assigned to the AB bond while only one third of the Type (b) and one half of the Type (c) can be so assigned.

Nearest-neighbor dependent bonds $\mathrm{AB}$ can be characterized by the number of atoms to which $\mathrm{A}$ is conjugated and the number to which $B$ is. For example, the 1-2,1-9, and 9-10 bonds in naphthalene (standard numbering) are examples of the $\pi^{2}-\pi^{2}, \pi^{2}-\pi^{3}$, and $\pi^{3}-\pi^{3}$ bonds, respectively. Table $V$ in the text was compiled by following the above counting rules and noting the numbers of the various arrangements in Fig. 6 that contribute to each bond.

\section{APPENDIX IV. BIPHENYL AND "BOND ENERGIES"}

If we omit compounds which merely differed in nonconjugated parts, the compounds considered by McGinn were the fused aromatics in Table $\mathrm{V}$, biphenyl, and a compound with similar conjugation, fluorene. ${ }^{23}$ Thus, we need consider only biphenyl and see how its apparent fit into a three-parameter family for additivity can be understood in terms of walks.

Each pair of fused rings was connected at two points and not by some third conjugated path in addition. In such fused-ring systems the relationship between the number of rings $N_{R}$ and the number of conjugated $\mathrm{CC}$ bonds $N_{C C}$ is the same as that for the linearly fused acenes: benzene, naphthalene, anthracene, etc.:

$$
N_{\mathrm{R}}=\frac{1}{5}\left(N_{\mathrm{CC}}-1\right) \text {. }
$$

Thus, if one adds to the fourth, fifth, and sixth rows of the last column of Table $V$ the amount $\frac{12}{5}$ (or slightly less, because of the -1 ), one obtains a fairly accurate count of all six-step walks, merely by counting the number of $\pi^{2}-\pi^{2}, \pi^{2}-\pi^{3}$, and $\pi^{3}-\pi^{3}$ bonds, as well as of the two-step and four-step walks. In the case of biphenyl, only a minor error in counting the number of circumnavigatory walks is introduced by

\footnotetext{
Graphite was also considered, but its difference from the fused aromatics only introduces another parameter, the relevant $\mathrm{C}-\mathrm{H}$ "bond energy," in this additivity.
} 
using the above correction numbers. Hence, the numbers of nearest-neighbor dependent bonds provides a way of counting numbers of two-step, four-step, and six-step walks, in the class of systems McGinn considered, so that the additivity behavior is expected. The additivity will begin to break down slightly when
Eq. (A1) is less applicable, for example in fused compounds such as pyrene, perylene and in vinyl compounds. An alternative formulation based on Table $\mathrm{V}$ might permit the inclusion of such compounds, if differences between ring bonds and bonds in polyenes are taken into account.

THE JOURNAL OF CHEMICAL PHYSICS V VLUME 43, NUMBER 8

\title{
On the Theory of Chemiluminescent Electron-Transfer Reactions*
}

\author{
R. A. Marcus \\ Noyes Chemical Laboratory, University of Illinois, Urbana, Illinois
}

(Received 7 May 1965)

\begin{abstract}
A mechanism is described for chemiluminescent electron-transfer reactions. It is shown that in the case of very exothermic homogeneous electron-transfer reactions, the intersection of the potential-energy surface of the reactants with that of electronically unexcited products occurs only at high energies. The rate of formation of unexcited products then becomes slow. A numerical estimate of this slowness is made using known homogeneous rate constants for ordinary electron-exchange reactions. An intersection occurs at lower energies when one of the products of a highly exothermic electron transfer is electronically excited, thereby reducing the exothermicity. The product may emit light or subsequently form a state that does.

A rather different situation is shown to occur at electrodes; the system can now reduce the "exothermicity" by having the electron transfer into a high unoccupied level of the conduction band or from a low occupied level of the latter. The large width of the conduction band in metals permits much latitude in reducing the exothermicity thereby.

These results are compared with present experimental findings that chemiluminescent electron transfers occur in solution rather than on electrode surfaces.
\end{abstract}

$\mathbf{A}^{\mathrm{v}}$ VARIETY of atom-transfer chemiluminescent reactions are known, but more recently a number of chemiluminescent electron-transfer reactions have been reported in the literature. ${ }^{\text {la }-d}$ We apply a theory of electron transfers ${ }^{2}$ to these reactions and examine some consequences for experiment.

We consider a potential-energy surface for the reactants and one for the products, each plotted as a function of all the translational, rotational, and vibrational coordinates in the system. ${ }^{2}$ In the zeroth approximation of no electronic coupling of the redox orbitals of the ion and electrode, these two potential-energy sur-

\footnotetext{
* Supported by a grant from the National Science Foundation. 1 (a) D. M. Hercules, Science 145, 808 (1964); (b) E. A. Chandross and F. I. Sonntag, J. Am. Chem. Soc. 86, 3179 (1964), and references contained therein; (c) K. S. V. Santhanan and A. J. Bard, ibid. 87, 139 (1965); (d) G. J. Hoitjink (private communication); (e) This possibility was suggested to the writer by G. J. Hoitjink: In some cases, polarographic data on aromatic compounds suggest that a reaction may be sufficiently exothermic to form a triplet state of an aromatic molecule but not quite enough to form an excited singlet. The triplets may then phosphoresce in rigid media, or in solution they may annihilate each other or react with other solutes. Polarographic data are summarized by $G$. J. Hoijtink, Ind. Chim. Belge 12, 1371 (1963).

2 (a) R. A. Marcus, Ann. Rev. Phys. Chem. 15, 155 (1964) and references cited therein; J. Chem. Phys. 43, 679 (1965); (b) Discussions Faraday Soc. 29, 21 (1960); (c) J. Phys. Chem. 67, 853,2889 (1963).
}

faces intersect. In the next approximation of some electronic coupling, the intersection is removed by the usual quantum-mechanical splitting, as in Fig. 1(a). A suitable fluctuation of coordinates involving approach of the reactants, reorientation of solvent molecules, and change in bond lengths of reactants, permits the system to cross the original intersection region in a coordinate region (small separation distance) where the electronic coupling is appreciable. In this way electron transfer has occurred, adiabatically if the splitting is sufficient and nonadiabatically otherwise. The system has moved from the $\mathrm{R}$ to the $\mathrm{P}$ surface [Fig. 1(a)].

In the usual thermal electron-transfer reaction, the products are formed in their electronic ground states, for only these are usually conveniently accessible energetically. Nevertheless, the potential-energy surface of the reactants will "cross" a surface of the products in which one or more products is electronically excited, in some other region of configuration space. If this latter intersection region is easily accessible (energetically and entropically), a reaction to form an excited product can occur. The excited product may either emit light or react. An example of such a reaction is a possible triplet-triplet annihilation to form an excited singlet state which later fluoresces. ${ }^{10}$ 